\title{
Descriptive temporal template features for visual motion recognition
}

\author{
Hongying Meng ${ }^{1}$ and Nick Pears ${ }^{2}$ \\ ${ }^{1}$ Department of Computing and Informatics, University of Lincoln, UK \\ ${ }^{2}$ Department of Computer Science, University of York, UK
}

\begin{abstract}
In this paper, a human action recognition system is proposed. The system is based on new, descriptive 'temporal template' features in order to achieve high-speed recognition in real-time, embedded applications. The limitations of the well known 'Motion History Image' (MHI) temporal template are addressed and a new 'Motion History Histogram' (MHH) feature is proposed to capture more motion information in the video. MHH not only provides rich motion information, but also remains computationally inexpensive. To further improve classification performance, we combine both MHI and MHH into a low dimensional feature vector which is processed by a support vector machine (SVM). Experimental results show that our new representation can achieve a significant improvement in the performance of human action recognition over existing comparable methods, which use $2 \mathrm{D}$ temporal template based representations.
\end{abstract}

Key words: Gesture recognition, Event recognition, Embedded vision, Motion analysis, Machine learning

\section{Introduction}

In this paper, we focus on the use of video for monitoring human events, with a view to real-time, embedded implementations in security systems, humancomputer interaction and intelligent environments. Such events may be prescribed gestures, or simple actions, such as walking in a particular direction across the scene. In particular, our methods are designed to be appropriate for deployment in a real-time, embedded context. In this sense, we aim for compact, yet descriptive image-based (2D) motion representations, which are simple to compute. Also we aim for low complexity classification algorithms, so that both feature extraction and classification may be implemented on our 
flexible stand-alone video processing architecture, which is based upon fieldprogrammable gate arrays (FPGAs). A detailed description of this hardware can be found in Meng et al. (2007a, 2008).

Generally, event detection in video has become an important computer vision application, particularly in the context of activity classification (Aggarwal and Cai (1999)). For human activity or behaviour recognition, many efforts have been concentrated on using state-space methods (Farmer et al. (1991)) to understand human motion sequences (Bobick and Davis (2001); Campbell and Bobick (1995); Gao et al. (2004); Nascimento et al. (2005); Davis and Tyagi (2006)). However, these methods usually need intrinsic nonlinear models and do not have a closed-form solution. Previous work on motion descriptors uses positions and velocities of human body parts (Green and Guan (2004)), but such information is often difficult to extract automatically during unrestricted human activities.

Aggarwal and Cai (1999) present an excellent overview of human motion analysis. Of the appearance based methods, template matching has gained increasing interest ( Schuldt et al. (2004); Weinland et al. (2005); Ke et al. (2005); Wong and Cipolla (2005); Dollár et al. (2005); Niebles et al. (2006); Ogata et al. (2006); Dalal et al. (2006); Blank et al. (2005); Oikonomopoulos et al. (2006); Meng et al. (2006b,a); Wong and Cipolla (2006); Yeo et al. (2006)). An example of a temporal template motion representation, the 'Motion History Image' (MHI) (Bobick and Davis (2001)), is given in Fig. 1, where (a) is one frame from the original video clip and (b) is the MHI of this action. It is clear that MHI is an image, with the same size as the original frame, but which retains motion information associated with the action. To compute an MHI, we start by computing a foreground motion segmentation, which may be done by simple frame differencing or more complex background modelling, such as Gaussian Mixture Models (Stauffer and Grimson (2000)). The MHI is then generated as the weighted sum of past foreground segmentations and the weights decay back through time. Therefore, an MHI image contains the past foreground segmentations within itself, where most recent foreground motion is brighter than past ones. (This is mathematically defined in section 3.)

We have elected to further develop this 'temporal-template' approach. However, given that the extracted motion information from temporal templates is appearance based, we have the limitations of (i) viewpoint dependence and (ii) loss of information in the projection from 3D to 2D. Both of these problems are mitigated if the motion is predominantly parallel to the image plane. In the case of gestures, these can be designed to be in an appropriate direction relative to the camera. In the context of less deliberative, natural human motion, the problem can only be solved by implementing multiple viewpoints and selecting the most appropriate data stream. This was a necessary compromise to allow us to implement a real-time embedded solution to our pattern 
recognition problem. Given that we have these restrictions, the problem that we wished to solve was how to extract more information in order to improve the state-of-the-art in temporal template based motion recognition approaches, while retaining a compact feature vector, that is easily deployed on embedded hardware.

In the following, we describe a human action recognition system based on a linear Support Vector Machine (SVM) (Cristianini and Shawe-Taylor (2000)). We address the limitations of the well-known Motion History Image (MHI) (Bobick and Davis (2001)), which is the pioneering work in temporal templates, and propose a new feature, which we call the Motion History Histogram $(\mathrm{MHH})$. This representation expresses more motion information than the MHI, but also remains inexpensive to compute. We show that the $\mathrm{MHH}$ and its derivatives outperform the MHI in terms of action recognition on a large public database. Finally, we extract a compact feature vector from the $\mathrm{MHH}$ and then combine it with a 'histogram of MHI' feature to give a better classification performance than either feature type alone.

The rest of this paper is organized as follows: In section 2, we give an overview of related work. In section 3, we briefly review the MHI and its limitation. In section 4, we give a detailed description of our new $\mathrm{MHH}$ temporal template features, designed to overcome the MHI limitation, and their compact derivatives. In section 5, we discuss dimension reduction and feature combination, applied to MHI and MHH. In section 6, experimental results are evaluated and, finally, we present conclusions. The work presented here is an extension of our previous work (Meng et al. (2006b, 2007b)).

\section{Related work}

Bobick and Davis (2001) pioneered the idea of temporal templates (Moeslund et al. (2006)). They used Motion Energy Images (MEI) and MHI to recognize many types of aerobics exercise. Bradski and Davis (2002) proposed the Motion Gradient Orientation (MGO) to explicitly encode changes in an image introduced by motion events.

Davis (2001) also presented a useful hierarchical extension for computing a local motion field from the original MHI representation. The MHI was transformed into an image pyramid, permitting efficient fixed-size gradient masks to be convolved at all levels of the pyramid, thus extracting motion information at a wide range of speeds. The hierarchical MHI approach remains a computationally inexpensive algorithm to represent, characterize, and recognize human motion in video. 
Schuldt et al. (2004) proposed a method for recognizing complex motion patterns based on local space-time features in video and they integrated such representations with SVM classification schemes for recognition.

The work of Efros et al. (2003) focuses on the case of low resolution video of human behaviours, targeting what they refer to as the 30 pixel man. In this setting, they propose a spatio-temporal descriptor based on optical flow measurements, and apply it to recognize actions in ballet, tennis and football datasets.

Weinland et al. (2005) introduced Motion History Volumes (MHV) as a freeviewpoint representation for human actions in the case of multiple calibrated, and background-subtracted, video. They presented algorithms for computing, aligning and comparing MHVs of different actions performed by different people from a variety of viewpoints.

Ke et al. (2005) studied the use of volumetric features as an alternative to the local descriptor approaches for event detection in video sequences. They generalized the notion of $2 \mathrm{D}$ box features to $3 \mathrm{D}$ spatio-temporal volumetric features. They constructed a real-time event detector for each action of interest by learning a cascade of filters based on volumetric features that efficiently scanned video sequences in space and time.

Ogata et al. (2006) proposed Modified Motion History Images (MMHI) and used an eigenspace technique to realize high-speed recognition of six human motions.

Wong and Cipolla (2005) proposed a new method to recognize primitive movements based on MGO extraction and, later, used it for continuous gesture recognition (Wong and Cipolla (2006)).

Recently, Dalal et al. (2006) proposed Histogram of Oriented Gradient (HOG) appearance descriptors for image sequences and developed a detector for standing and moving people in video.

Dollár et al. (2005) proposed a similar method where they use a new spatiotemporal interest point detector to obtain a global measurement instead of the local features in Efros et al. (2003). Niebles et al. (2006) also use spatial-time interest points to extract spatial-temporal words as their features. Yeo et al. (2006) estimate motion vectors from optical flow and calculate frame-to-frame motion similarity to analyse human action in video.

Blank et al. (2005) regarded human actions as three dimensional shapes induced by silhouettes in space-time volume. They adopted an approach for analyzing 2D shapes and generalized it to deal with volumetric space-time action shapes. 
Oikonomopoulos et al. (2006) introduced a sparse representation of image sequences as a collection of spatio-temporal events that were localized at points that were salient both in space and time for human action recognition.

We note that, in some of these methods, the motion features employed are relatively complex (Efros et al. (2003); Schuldt et al. (2004); Weinland et al. (2005); Niebles et al. (2006); Dalal et al. (2006); Dollár et al. (2005); Blank et al. (2005); Oikonomopoulos et al. (2006); Ke et al. (2005); Yeo et al. (2006)), which implies significant computational cost when building the features. Some of them require tracking or other prohibitive computational cost processes (Bobick and Davis (2001); Bradski and Davis (2002); Davis (2001); Wong and Cipolla (2005, 2006); Ogata et al. (2006); Blank et al. (2005)), which, for the time being, makes them not suitable for real-time embedded vision applications.

\section{The Motion History Image}

Several representations have been proposed to compact the whole motion sequence of a video clip into one image to represent the motion. Such images are often termed 'temporal templates'. The most popular 'temporal template' motion feature is the Motion History Image (MHI) (Bobick and Davis (2001)). We now review this in outline and then we discuss its main limitation, that we aim to overcome, by design of a new feature type.

An MHI is the weighted sum of past images and the weights decay back through time. Therefore, an MHI image contains the past images within itself, in which the most recent image is brighter than past ones. Normally, an MHI $H_{\tau}(u, v, k)$ at time $k$ and location $(u, v)$ is defined by the following Eq. 1:

$$
H_{\tau}(u, v, k)=\left\{\begin{array}{l}
\tau, \quad \text { if } D(u, v, k)=1 \\
\max \left\{0, H_{\tau}(u, v, k-1)-1\right\}, \text { otherwise }
\end{array}\right.
$$

where the motion mask $D(u, v, k)$ is a binary image representing the foreground motion, obtained from subtraction of frames, and $\tau$ is the maximum duration a motion is stored. In general, $\tau$ is chosen as the constant 255 , allowing the MHI to be easily represented as a gray scale image with one byte depth. Thus an MHI pixel can have a range of values, whereas an MEI (motion energy image) is its binary version, that can easily be computed by thresholding $H_{\tau}>0$.

Fig. 1 shows an example of the MHI motion features. Although we do not see the original video clip of the action, we can still determine that the subject 


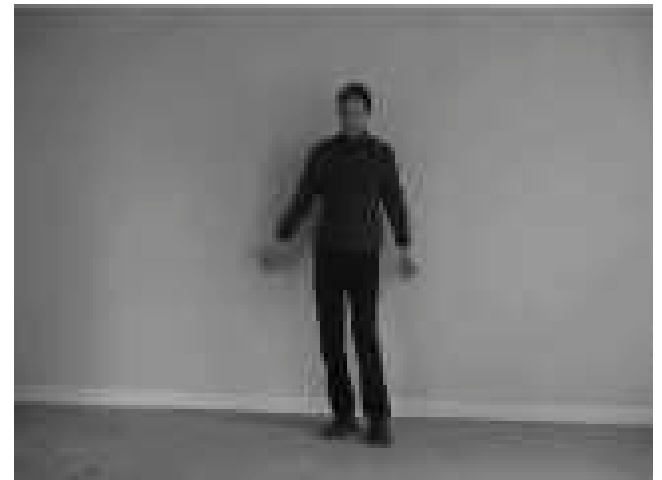

(a) Handwaving sample

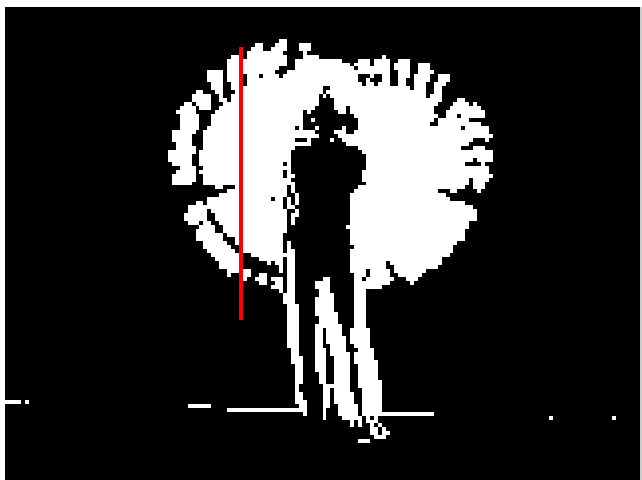

(b) MHI of Handwaving

Fig. 1. Example of an MHI. (a) is one frame from the original hand waving action video clip and (b) is the MHI of this action. The vertical red line in (b) has the pixels from $(60,11)$ to $(60,80)$.

moved both arms above their head. An MHI can be generated frame by frame and only three frames (previous frame, current frame and MHI) need to be stored at any one time. The final output associated with each action is an image, which has the same size as the original input frames.

\subsection{Limitations of the $M H I$}

In order to have a detailed look at the MHI, we have selected the pixels on the red line in the MHI of Fig. 1 (b). If some action happened at frame $k$ on pixel $(u, v)$, then $D(u, v, k)=1$, otherwise $D(u, v, k)=0$. The locations of these pixels are $(60,11),(60,12), \cdots,(60,80)$. For a pixel $(u, v)$, the motion mask $D(u, v,:)$ of this pixel is the binary sequence:

$$
D(u, v,:)=\left(b_{1}, b_{2}, \cdots, b_{N}\right), \quad b_{i} \in\{0,1\}
$$

where $N+1$ is the total number of frames (as we need two images at the start of the sequence to generate $b_{1}$ ).

All of the motion masks on the red line are shown in Fig. 2. Each row is $D(u, v$, : ) for one fixed pixel $(u, v)$ and a white block represents ' 1 ' and black block represents ' 0 ' in the sequences. The green line is the motion mark $D(60,50,:)$ and it has the following sequence (3):

$$
0000000001101000000000000000000000001010000
$$

From the definition of MHI in Eq. 1 it can be observed that, for each pixel $(u, v)$, MHI actually retains the time since the last action occurred. That is, only the last ' 1 ' in the sequence 3 is retained in the MHI at pixel $(60,50)$. 


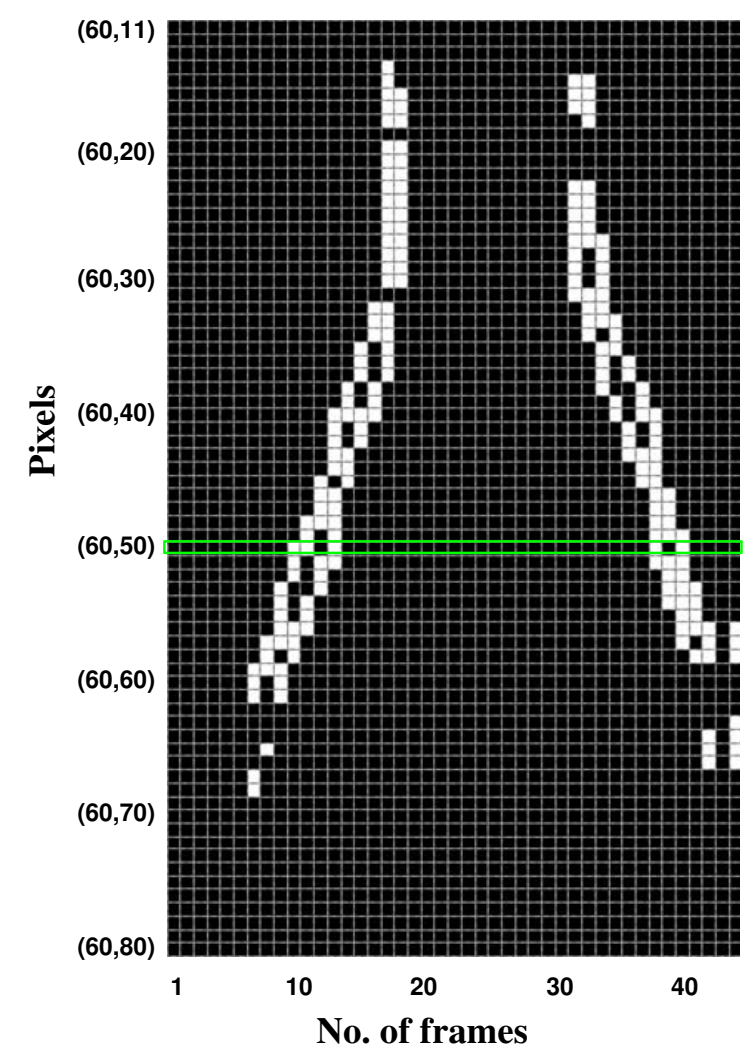

Fig. 2. $D(:,:,:)$ on the red line of Fig. 1(b) is shown. Each row is $D(u, v,:)$ for one fixed pixel $(u, v)$. A white block represents ' 1 ' and a black block ' 0 '. The horizontal green line is the 'binary frame difference history' or 'motion mask' of pixel $(60,50)$ through time, ie, $D(60,50,:)$.

It is clear that previous ' 1 's in the sequence, when some action occurred, are not represented. It is also clear that almost all the pixels have more than one ' 1 ' in their sequence, implying that much motion information is lost when an MHI is generated.

\section{Motion History Histograms (MHH)}

The above limitation of the MHI has motivated us to design a new representation (the MHH) in which all of the information in the sequence is used and, yet, it remains compact and efficient to use.

We define the patterns $P_{i}$ in the $D(u, v,:)$ sequences, based on the number of 


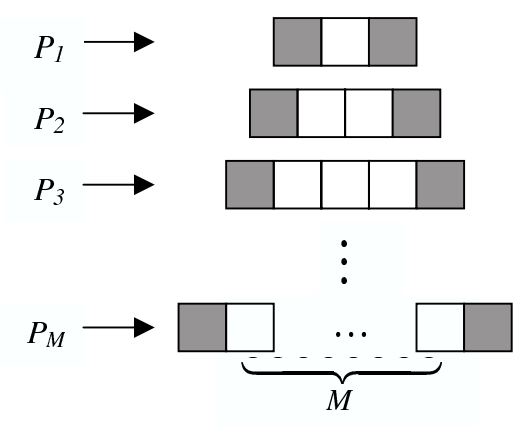

Fig. 3. The patterns $P_{i}$ in $D(u, v,:)$. White and black blocks represent '1' and ' 0 ' respectively.

connected '1's, as shown in Fig. 3:

$$
\begin{gathered}
P_{1}=010 \\
P_{2}=0110 \\
P_{3}=01110 \\
\vdots \\
P_{M}=0 \underbrace{1 \cdots 1}_{M} 0
\end{gathered}
$$

We denote a subsequence $C_{I, k}$ by Eq. 5 , where $I$ and $k$ are the indices of starting and ending frames, and denote the set of all subsequences of $D(u, v,:)$ as $\Omega\{D(u, v,:)\}$. Then, for each pixel $(u, v)$, we can count the number of occurrences of each specific pattern $P_{i}$ in the sequence $D(u, v,:)$, as shown in Eq. 6, where $\mathbf{1}$ is the indicator function.

$$
\begin{gathered}
C_{I, k}=b_{I}, b_{I+1}, \cdots, b_{k}, \quad 1 \leq I<k \leq N \\
M H H(u, v, i)=\sum_{(I, k)} \mathbf{1}_{\left\{\mathbf{C}_{\mathbf{I}, \mathbf{k}}=\mathbf{P}_{\mathbf{i}} \mid \mathbf{C}_{\mathbf{I}, \mathbf{k}} \in \boldsymbol{\Omega}\{\mathbf{D}(\mathbf{u}, \mathbf{v},:)\}\right\}} \\
1 \leq I<k \leq N, 1 \leq i \leq M
\end{gathered}
$$

From each pattern $P_{i}$, we can build a gray scale image and we call this its 'Histogram', since the bin value records the number of this pattern type. With all the patterns $P_{i}, i=1 \ldots M$ together, we collectively call them the 'Motion History Histograms'(MHH) representation.

The computation of $\mathrm{MHH}$ is inexpensive and can be implemented by the following procedure. $D(u, v, k)$ is the binary sequences on pixel $(u, v)$ that is computed by thresholding the differences between frame $k$ and frame $k-1$. $I(u, v)$ is a frame index that stands for the number of the starting frame of a new pattern on pixel $(u, v)$. At the beginning, $I(u, v)=1$ for all $(u, v)$. 


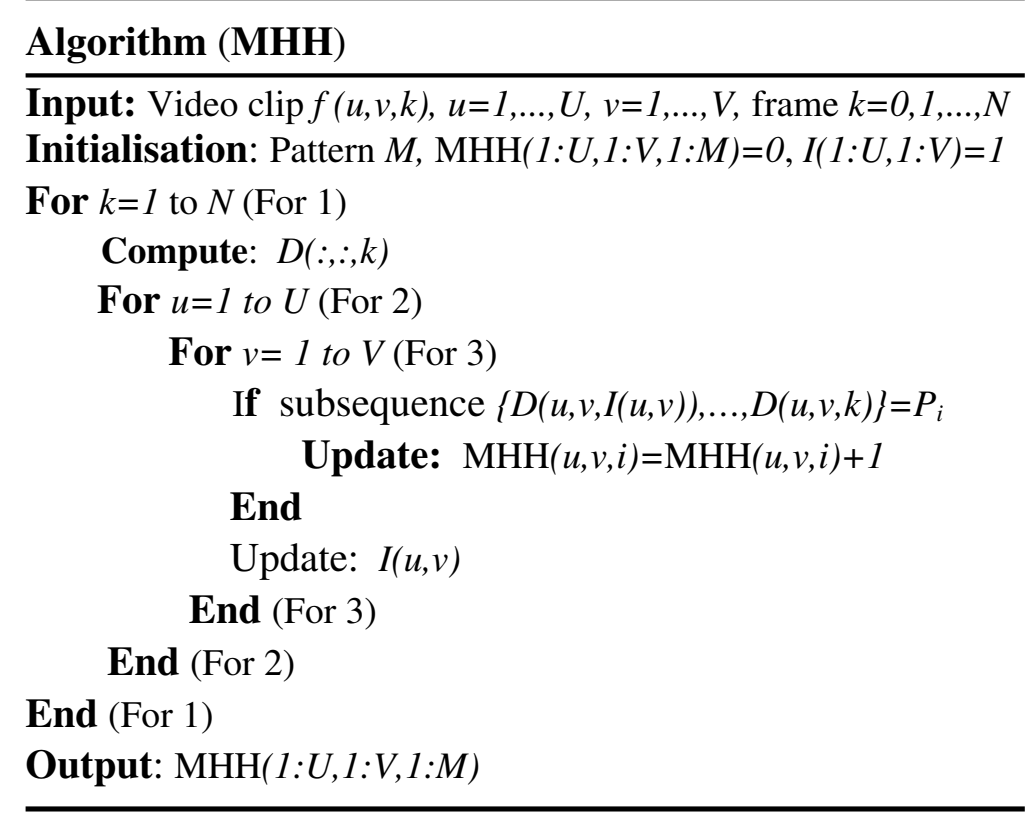

That means a new pattern starts from frame 1 for every pixel. $I(u, v)$ will be updated to $I(u, v)=k$ while $\{D(u, v, I(u, v)), \ldots, D(u, v, k)\}$ builds one of the patterns $P_{i}(1 \leq i \leq M)$ and, in this case, $\operatorname{MHH}(u, v, i)$ increases by 1 .

For a pattern $P_{i}, \operatorname{MHH}(:,:, i)$ can be displayed as an image. In Fig. 4, four patterns $P_{1}, P_{2}, P_{3}$ and $P_{4}$ are shown, which were generated from the hand waving action in Fig. 1. By comparing the MHH in Fig. 4 with the MHI in Fig. 1, it is interesting to find that the $\mathrm{MHH}$ decomposes the MHI into different parts based on patterns. Unlike the hierarchical MHI described by Davis (2001), where only small size MHIs were obtained, MHH records the rich spatial information of an action.

The choice of the number $M$ depends on the video clips. In general, the bigger the $M$ is, the better the motion information will be. However, the values within the $\mathrm{MHH}$ rapidly approach zero as $M$ increases. In our experiment, no more than half of the training data had the sixth pattern $P_{6}$ and so we chose $M=5$. Furthermore we note that a large $M$ will increase the storage requirement for our hardware based system.

We can define the binary version of an $\mathrm{MHH}$ as $\mathrm{MHH}_{b}$, as shown in Eq. 7, and the motivation for this is given in the following section.

$$
M H H_{b}(u, v, i)=\left\{\begin{array}{l}
1, \text { if } M H H(u, v, i)>0 \\
0, \text { otherwise }
\end{array}\right.
$$




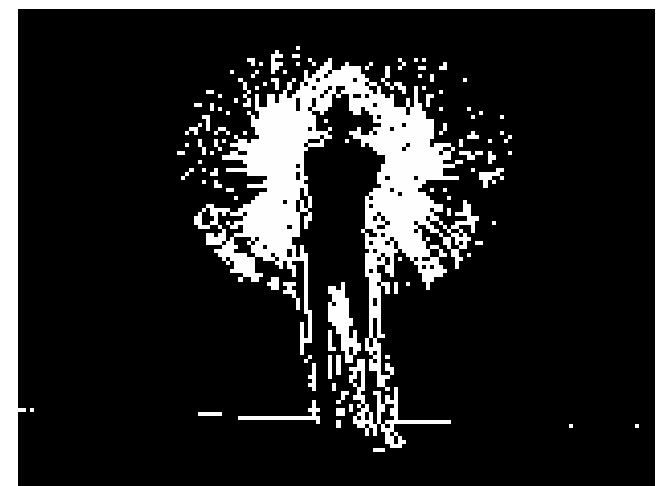

(a) $\mathrm{MHH}(:,:, 1)$

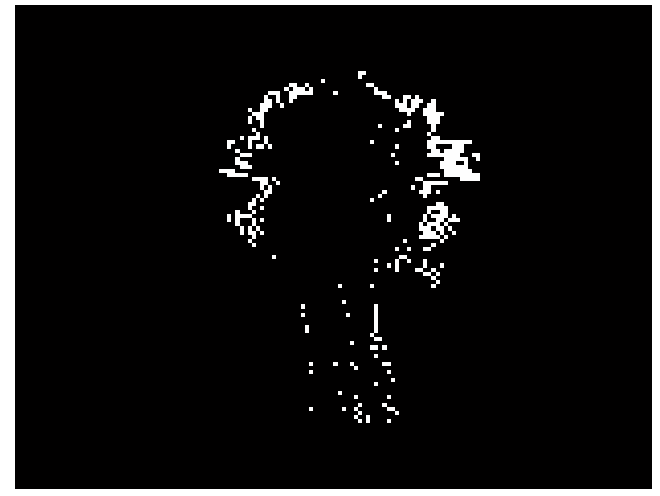

(c) $\mathrm{MHH}(:,:, 3)$

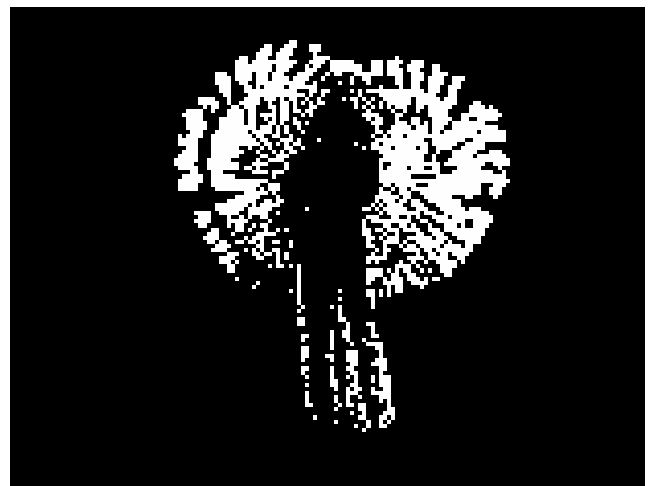

(b) $\mathrm{MHH}(:,:, 2)$

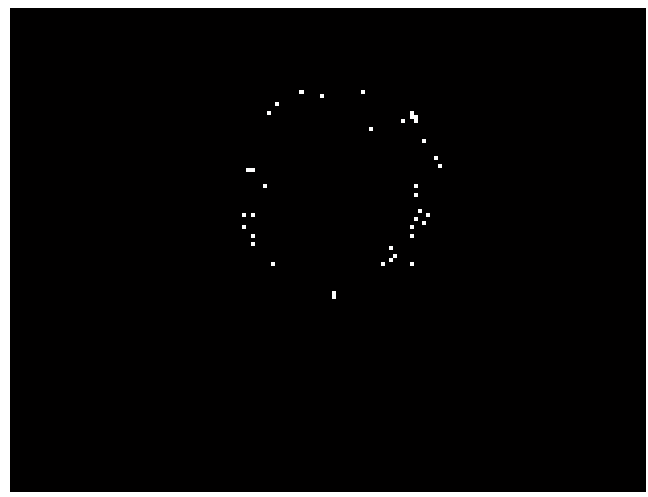

(d) $\mathrm{MHH}(:,:, 4)$

Fig. 4. MHH example. Four patterns $P_{1}, P_{2}, P_{3}$ and $P_{4}$ were selected. This results were generated from the handwaving action in Fig. 1. Each pattern $P_{i}, \operatorname{MHH}(:,:, i)$ has the same size as the original frame.

\section{Dimension reduction and feature combination}

As previously stated, our work is targeted at embedded implementations using FPGA platforms. This requires us to use compact feature vectors, which retain discriminating information across the various classes of interest in a particular application. Also, the process of compacting must be simple, in order to generate a small footprint (gate count) solution on our embedded devices. The following subsections describe how we employ histograms of both MHI and MHH representations, to compact their representations, and then combine them via simple feature vector concatenation.

\subsection{MHI Histograms}

MHIs can be rendered as gray scale images, where a value of a pixel in the MHI records time information, namely when some motion most recently occurred at this particular pixel location. Thus the histogram of MHI captures information 
representing the speed of the motion across the image. Since an MHI is an image with values between 0 and 255, an MHI histogram is a vector of length 256. An example of an MHI histogram is given at the bottom-left of Fig. 5. For fast motions, many pixels in the MHI will have high values, whereas, for slow motions, only a few pixels in the MHI will have high values, thus these different cases will have quite different MHI histograms.

\subsection{MHH Histograms : the Motion Geometric Distribution (MGD)}

The size of the $\mathrm{MHH}_{b}$ representation is rather large and we seek a more compact representation, which captures the geometric distribution of the motion across the image. Thus we sum each row of $\mathrm{MHH}_{b}$ (for a given pattern, $P_{i}$ ) to give a vector of size $V$ rows. We obtain another vector by summing columns to give a vector of size $U$ rows. Thus using all $M$ levels in the binarised MHH hierarchy, we obtain a 'Motion Geometric Distribution' (MGD) vector of size $M \times(U+V)$, which is relatively compact, when compared to the size of the original MHH and MHI features. The MGD vector can thus be represented by the following Eq. 8:

$$
\begin{gathered}
M G D=\left\{\sum_{u} M H H_{b}(u, v, i), \sum_{v} M H H_{b}(u, v, i)\right\} \\
i=1,2, \cdots, M
\end{gathered}
$$

In our work, we prefer to compute the MGD by using the $\mathrm{MHH}_{b}$ feature instead of the MHH feature directly. From our experiments, it has been found that the values within the $\mathrm{MHH}$ decrease significantly for the large patterns. The values for $P_{4}$ and $P_{5}$, for example, are much smaller than those of $P_{1}, P_{2}$ and $P_{3}$. Thus, if we use the MHH directly to compute the MGD, a normalisation process is necessary in order to treat all the patterns equally. However, this normalisation process is not an easy task for our hardware implementation because of limited memory and the requirement to implement a floating-point processing ability. In contrast, computation of the MGD from the $\mathrm{MHH}_{b}$ feature does not need a normalisation process and yet we are able to retain a good performance.

\subsection{Combining $M H H$ and MHI histogram features}

If different features are able to capture different discriminating properties of a video clip, then a combination of such features is likely to improve classification performance over either feature alone. Indeed, this is what we find in the following section, which describes our evaluation. Based on the simplicity requirement of our embedded systems, our two feature vectors are combined 

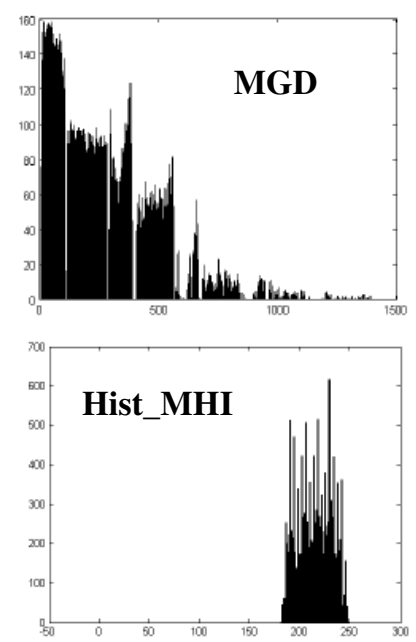
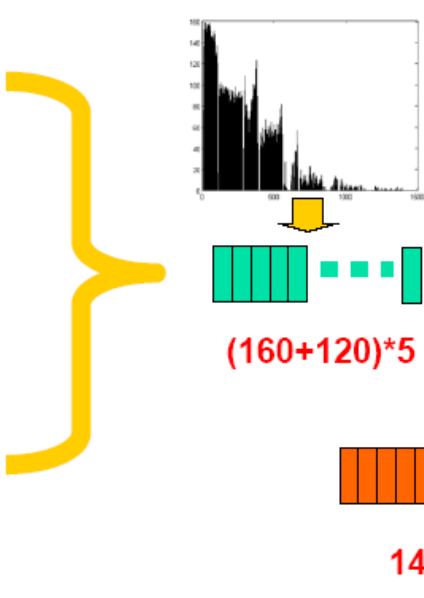

$(160+120)^{* 5}$
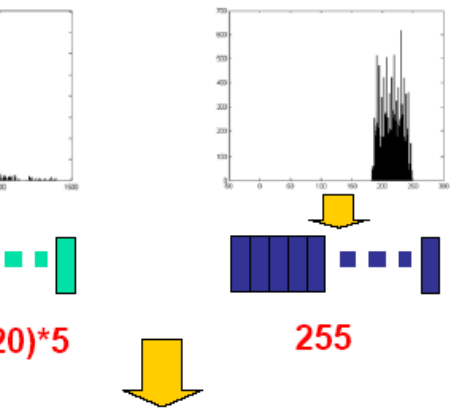

255

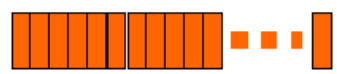

$1400+255=1655$

Fig. 5. Combination between MGD of the MHH and histogram of the MHI from a same video example. The frame has the size of $160 \times 120$. MGD of MHH and histogram of MHI have the size of $(160+120) \times 5=1400$ and 255 respectively.

in the simplest way by concatenating these two feature vectors into a higher dimensional vector. Fig. 5 shows an example of a combination between the MGD of the MHH and the histogram of the MHI from the same video.

\section{Evaluation of our system}

\subsection{Experimental setup}

For the evaluation of our system, we use a challenging human action recognition database, recorded by Schuldt et al. (2004), which is both large and publically available. It contains six types of human actions (walking, jogging, running, boxing, hand-waving and hand-clapping) performed several times by 25 subjects in four different scenarios: outdoors (s1), outdoors with scale variation (s2), outdoors with different clothes (s3) and indoors (s4).

This database contains 2391 sequences. All sequences were taken over homogeneous backgrounds with a static camera with $25 \mathrm{~Hz}$ frame rate. The sequences were down-sampled to the spatial resolution of $160 \times 120$ pixels and have a time length of four seconds in average. To the best of our knowledge, this is the largest video database with sequences of human actions taken over different scenarios. All sequences were divided with respect to the subjects into a training set ( 8 persons), a validation set ( 8 persons) and a test set (9 persons).

In our experiment, the classifiers were trained on a training set while classification results were obtained on the test set. In all our experiments, the 
(a)
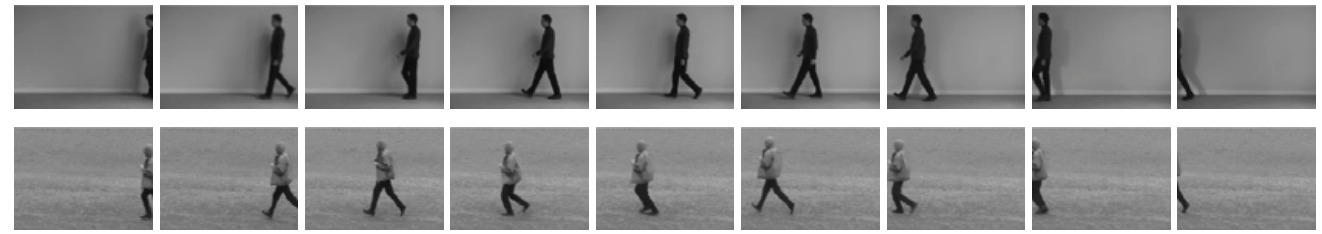

(c)
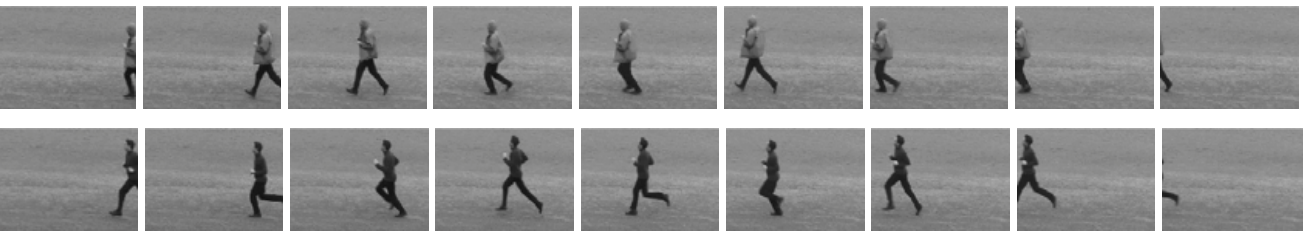

(d)
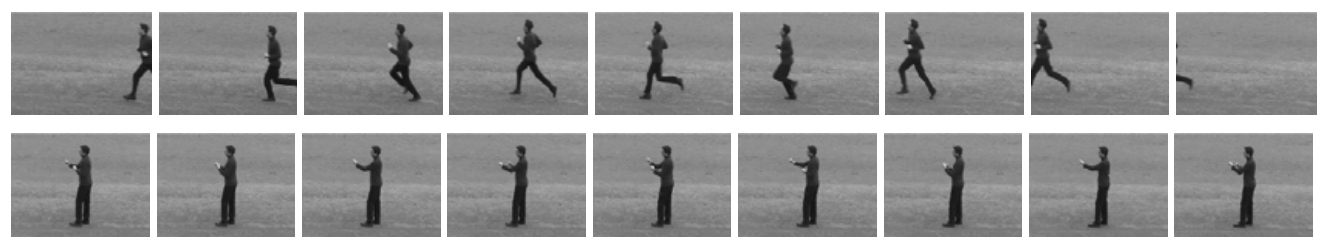

(e)
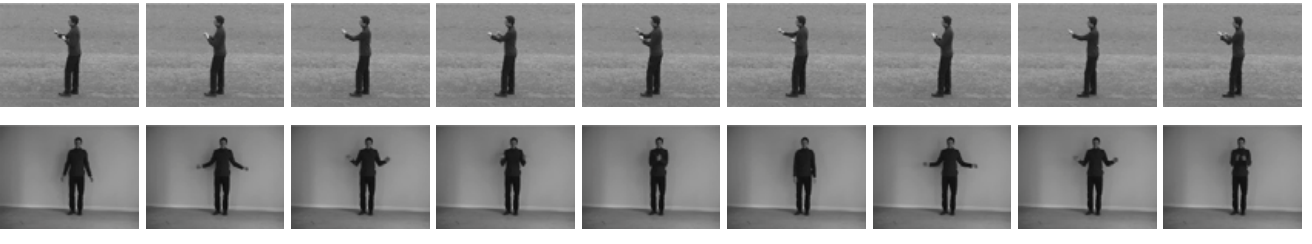

(f)
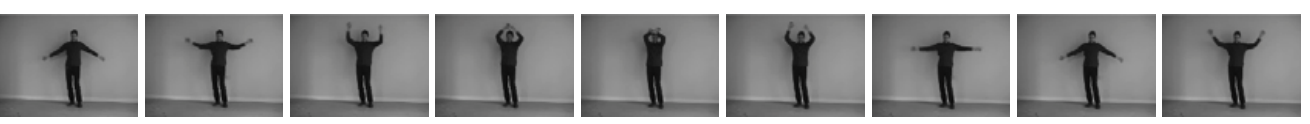

Fig. 6. Six types of human action in the database: (a) walking (b) jogging (c) running (d) boxing (e) hand-clapping (f) hand-waving.

same parameters were used. The threshold in frame differencing was chosen as 25 and $\tau$ was chosen as 255 for MHI construction. The most suitable choice of the number of patterns $M$ for $\mathrm{MHH}$ computation depends on the video clips and is a tradeoff between the compactness of the representation and the expressiveness of the representation. Building a frequency histogram of the patterns extracted from the training clips indicates that no more than half of the training data had the sixth pattern. Thus the number of patterns was chosen to be $M=5$.

The size of the MHI is $160 \times 120=19200$, which is the same width as that of the frames in the videos. In our experiment, the SVM is implemented using the $S V M^{\text {light }}$ software (Joachims (1999)). In SVM training, choosing a good parameter $C$ value is not so straightforward and can significantly affect classification accuracy (Hastie et al. (2004)) but in order to keep our system simple, the default value of $C$ in $S V M^{\text {light }}$ is used in all the experiments.

Fig. 6 shows examples in each type of human action in this dataset. In order to compare our results with those in Ke et al. (2005) and Schuldt et al. (2004), we use the exact same training set and testing set in our experiments. The only difference is that we did not use the validation dataset in training. Our experiments are carried out on all four different scenarios. In the same manner as Ke et al. (2005), each sequence is treated individually during the training and classification process. In all the following experiments, the parameters were are the same. 
(1)

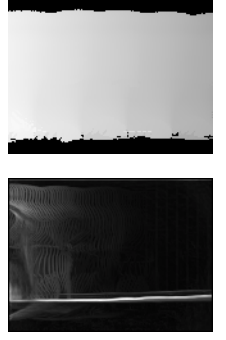

(3)

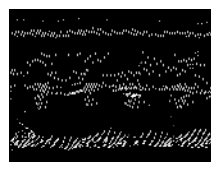

(a)
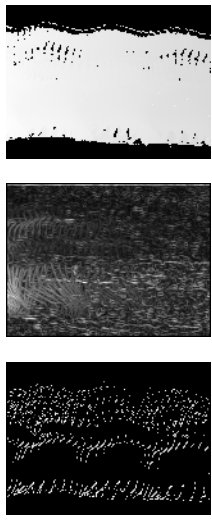

(b)
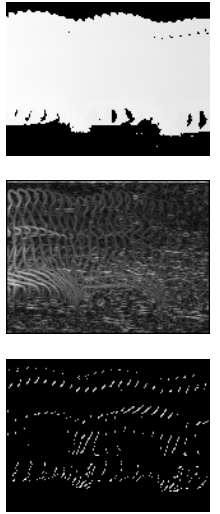

(c)
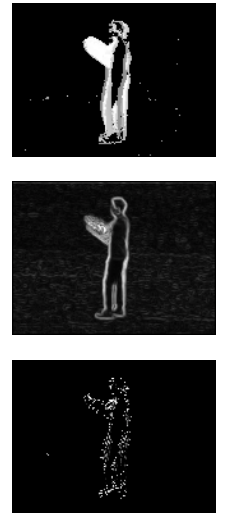

(d)
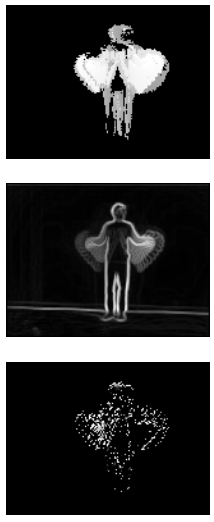

(e)
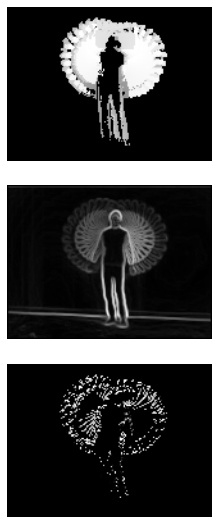

(f)

Fig. 7. The (1) MHI, (2) MMHI and (3) MGO for the six actions in the dataset: (a) walking (b) jogging (c) running (d) boxing (e) hand-clapping (f) hand-waving

\subsection{Performance on single features}

We have tested the performance of the fundamental motion features MHI, MMHI and MGO in our system. Fig. 7 shows these three motion features extracted from the action examples shown in Fig. 6. In order to keep our system simple for hardware implementation, we use the simplest method to transform the motion features (MHI, MMHI and MGO) into a plain vector based on the pixel scan order (row by row) to feed the SVM classifier.

Firstly, we tested the system performance on the four different subsets of the whole dataset. The results can be seen in Fig. 8. The correctly classified percentage on these data subsets indicates how many percent of the action clips in the testing set were correctly recognized by the system. It is clear that the MHI feature gave the best classification performance in all the four subset while the MGO feature gave poor results for all four data subsets. We also can see that subset s2 (outdoors with scale variation) is the most difficult subset in the whole dataset.

From the experiments, it can be seen that this type of system can get reasonable results. The MHI based system looks better than the MMHI system in the experiments. The disadvantage for MMHI is that it can only work well in the case of an uncluttered and static background. If there is background motion or noise, this will be recorded in the feature vector and will reduce the performance of the classification.

For the whole dataset, the classification confusion matrix is a good measure for the overall performance in this multi-class classification problem. Table 1 shows the classification confusion matrix based on the method proposed in Ke et al. (2005). Table 2 shows the confusion matrix obtained by our system based on MHI. The confusion matrices show the motion label (vertical) versus 


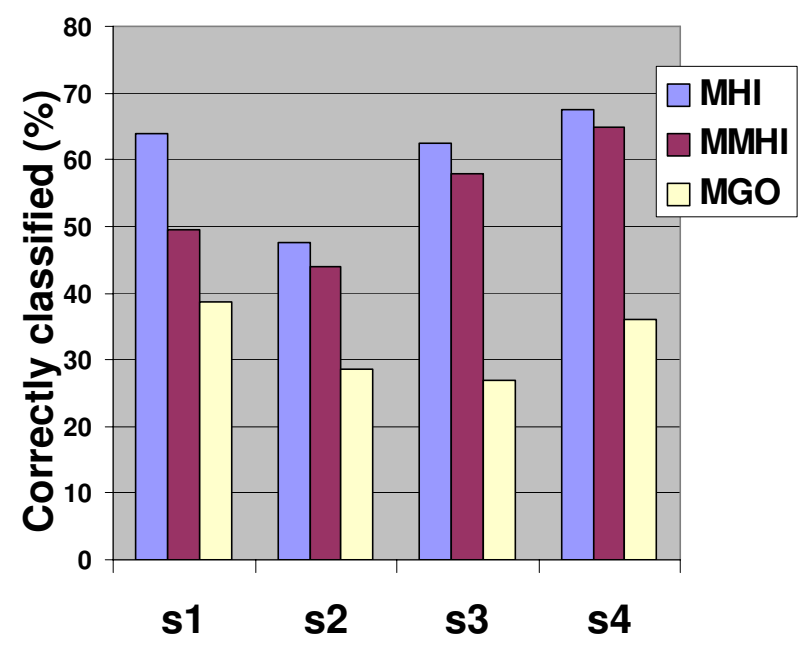

Fig. 8. Correctly classified percentage for separate data subset: s1 (outdoors), s2 (outdoors with scale variation), s3 (outdoors with different clothes) and s4 (indoors).

the classification results (horizontal). Each cell $(i, j)$ in the table shows the percentage of class $i$ action being recognized as class $j$. Thus the main diagonal of the matrices show the percentage of correctly recognized actions, while the remaining cells show the percentages of misclassification. The trace of the matrix shows the overall classification rate. In Table 1, the trace is 377.8 and since there are six classes, then the overall mean classification rate is $377.8 / 6=63 \%$.

Table 1

Ke's confusion matrix (Ke et al. $(2005)$ ), trace $=377.8$, mean performance $=63 \%$

\begin{tabular}{ccccccc}
\hline & Walk & Jog & Run & Box & Clap & Wave \\
\hline Walk & $\mathbf{8 0 . 6}$ & 11.1 & 8.3 & 0.0 & 0.0 & 0.0 \\
Jog & 30.6 & $\mathbf{3 6 . 2}$ & 33.3 & 0.0 & 0.0 & 0.0 \\
Run & 2.8 & 25.0 & $\mathbf{4 4 . 4}$ & 0.0 & 27.8 & 0.0 \\
Box & 0.0 & 2.8 & 11.1 & $\mathbf{6 9 . 4}$ & 11.1 & 5.6 \\
Clap & 0.0 & 0.0 & 5.6 & 36.1 & $\mathbf{5 5 . 6}$ & 2.8 \\
Wave & 0.0 & 5.6 & 0.0 & 2.8 & 0.0 & $\mathbf{9 1 . 7}$ \\
\hline
\end{tabular}

In comparison with Ke's method, we use a simple MHI feature rather than large volumetric features in which the dimension of a feature vector might be a billion, yet the performance of our system is marginally better on this dataset. Notice from Table 2 that the actions of walking, jogging and running are not well discriminated, as they are similar actions, performed at different speeds, whereas the more distinctive actions of boxing, hand-waving and handclapping appear to be easier to classify.

In a second series of experiments, we tested low dimensional features, which 
Table 2

MHI's confusion matrix, trace $=381.2$, mean performance $=63.5 \%$

\begin{tabular}{ccccccc} 
& Walk & Jog & Run & Box & Clap & Wave \\
\hline Walk & $\mathbf{5 3 . 5}$ & 27.1 & 16.7 & 0.0 & 0.0 & 2.8 \\
Jog & 46.5 & $\mathbf{3 4 . 7}$ & 16.7 & 0.7 & 0.0 & 1.4 \\
Run & 34.7 & 28.5 & $\mathbf{3 6 . 1}$ & 0.0 & 0.0 & 0.7 \\
Box & 0.0 & 0.0 & 0.0 & $\mathbf{8 8 . 8}$ & 2.8 & 8.4 \\
Clap & 0.0 & 0.0 & 0.0 & 7.6 & $\mathbf{8 7 . 5}$ & 4.9 \\
Wave & 0.0 & 0.0 & 0.0 & 8.3 & 11.1 & $\mathbf{8 0 . 6}$ \\
\hline
\end{tabular}

Table 3

MHI_S's confusion matrix, trace $=377.7$, mean performance $=62.95 \%$

\begin{tabular}{ccccccc}
\hline & Walk & Jog & Run & Box & Clap & Wave \\
\hline Walk & $\mathbf{5 6 . 9}$ & 18.1 & 22.2 & 0.0 & 0.0 & 2.8 \\
Jog & 45.1 & $\mathbf{2 9 . 9}$ & 22.9 & 1.4 & 0.0 & 0.7 \\
Run & 34.7 & 27.8 & $\mathbf{3 6 . 1}$ & 0.0 & 0.0 & 1.4 \\
Box & 0.0 & 0.0 & 0.0 & $\mathbf{8 9 . 5}$ & 2.1 & 8.4 \\
Clap & 0.0 & 0.0 & 0.0 & 5.6 & $\mathbf{8 8 . 9}$ & 5.6 \\
Wave & 0.0 & 0.0 & 0.0 & 12.5 & 11.1 & $\mathbf{7 6 . 4}$ \\
\hline
\end{tabular}

Table 4

Hist. of MHI's confusion matrix, trace $=328.6$, mean performance $=54.8 \%$

\begin{tabular}{ccccccc}
\hline & Walk & Jog & Run & Box & Clap & Wave \\
\hline Walk & $\mathbf{6 2 . 5}$ & 32.6 & 0.0 & 1.4 & 1.4 & 2.1 \\
Jog & 12.5 & $\mathbf{5 8 . 3}$ & 25.0 & 0.0 & 0.0 & 4.2 \\
Run & 0.7 & 18.8 & $\mathbf{7 7 . 1}$ & 0.0 & 0.0 & 3.5 \\
Box & 4.9 & 2.8 & 0.7 & $\mathbf{1 7 . 5}$ & 61.5 & 12.6 \\
Clap & 4.9 & 2.1 & 0.7 & 11.1 & $\mathbf{7 5 . 0}$ & 6.3 \\
Wave & 5.6 & 3.5 & 6.9 & 20.1 & 25.7 & $\mathbf{3 8 . 2}$ \\
\hline
\end{tabular}

are generated from fundamental motion features, such as MHI. Sub-sampling is easy to implement in hardware by any factor of 2 and this can be done in both rows and columns of the motion feature. Tables 3 and 4 show the results based on downsampling the MHI by a factor of 64 (a factor of 8 for both row and column) and the histogram of (subsampled) MHI respectively. It can be seen that subsampling the MHI to a significantly lower resolution does not have an unduly detrimental effect on classification performance. This feature 
(a)

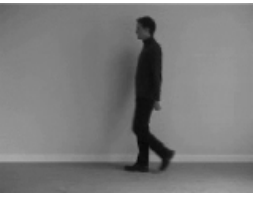

(b)

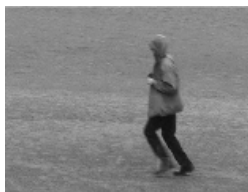

(c)

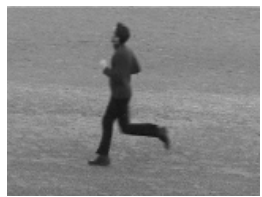

(d)

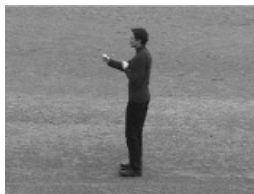

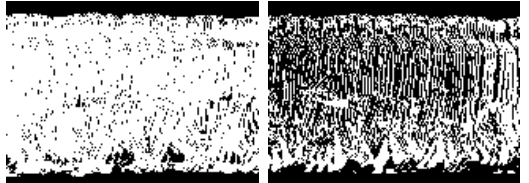
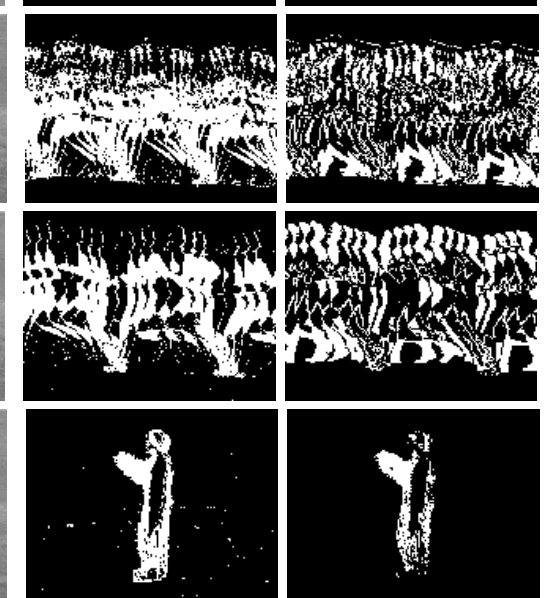

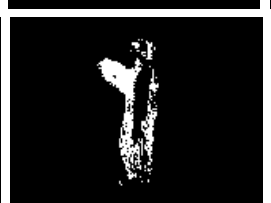

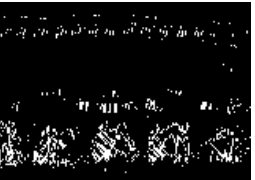

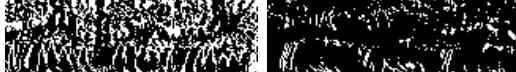

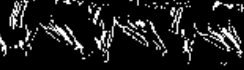
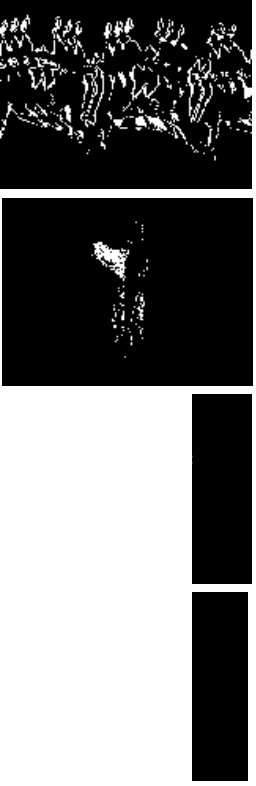

3)
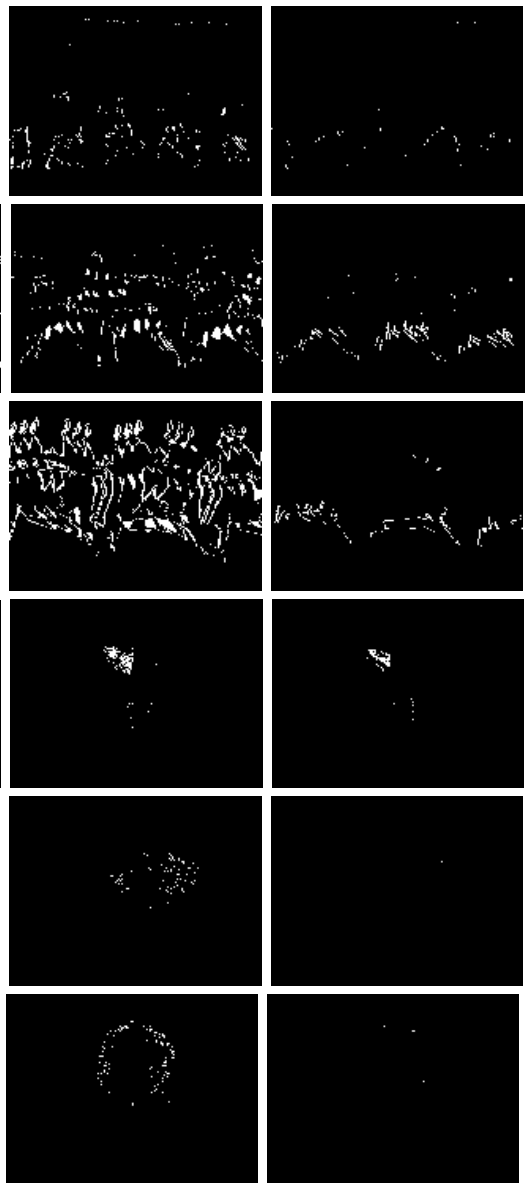

(5) $\operatorname{MHH}(:,:, 4)$

(6)MHH(:,:,5)

Fig. 9. The six database human actions and associated MHH features: (a) walking (b) jogging (c) running (d) boxing (e) hand-clapping (f) hand-waving.

performed well in distinguishing the last three groups (box, clap, wave). On the other hand, the histogram of MHI did not perform well in terms of overall performance but has the power to distinguish the first three groups of action (walk, jog, run), which are similar shaped actions, executed at significantly different speeds. This demonstrates that these two features make different types of information more explicit to the classification process and this provides a strong motivation for combining different feature types.

Fig. 9 shows examples in each type of human action and their associated MHH motion features. For the MHH, it is hard to deal with the whole feature in our target hardware system as, with the number of patterns set to 5 , the $\mathrm{MHH}$ has a relatively high dimension of $5 \times 160 \times 120=96000$. Thus, we constructed a smaller sized MHH_S by averaging the pixels in an $8 \times 8$ block, so that the size of all MHH feature vectors is reduced to $20 \times 15 \times 5=1500$. Our MGD feature also has a small size of $(160+120) \times 5=1400$.

Table 5 and Table 6 show the results when using features MHH_S and MGD respectively. From these two tables, it is very clear that both MHH_S and MGD improve the overall performance, but they are failed to classify the 'jogging' class. The reason is that these video clips are quite similar to walking and running. It is hard to distinguish them correctly even by human observation. This motivates us to consider the combination of the MGD with a feature that is particularly sensitive to the speed of the motion, as is the case with the 'histogram of MHI'. 
Table 5

MHH_S's confusion matrix, trace $=417.3$, mean performance $=69.55 \%$

\begin{tabular}{ccccccc} 
& Walk & Jog & Run & Box & Clap & Wave \\
\hline Walk & $\mathbf{8 8 . 9}$ & 1.4 & 6.3 & 0.7 & 1.4 & 1.4 \\
Jog & 56.9 & $\mathbf{2 . 1}$ & 38.2 & 0.7 & 2.1 & 0.0 \\
Run & 22.2 & 0.7 & $\mathbf{7 5 . 7}$ & 0.0 & 1.4 & 0.0 \\
Box & 0.0 & 0.0 & 0.0 & $\mathbf{9 6 . 5}$ & 0.7 & 2.8 \\
Clap & 0.0 & 0.0 & 0.0 & 4.2 & $\mathbf{9 3 . 1}$ & 2.8 \\
Wave & 0.0 & 0.0 & 0.0 & 22.2 & 16.7 & $\mathbf{6 1 . 1}$ \\
\hline
\end{tabular}

Table 6

MGD's confusion matrix, trace $=432.6$, mean performance $=72.1 \%$

\begin{tabular}{ccccccc} 
& Walk & Jog & Run & Box & Clap & Wave \\
\hline Walk & $\mathbf{8 5 . 4}$ & 4.9 & 2.8 & 2.8 & 2.8 & 1.4 \\
Jog & 65.3 & $\mathbf{9 . 2}$ & 23.6 & 2.1 & 0.0 & 0.0 \\
Run & 18.8 & 8.3 & $\mathbf{6 8 . 8}$ & 1.4 & 0.0 & 2.8 \\
Box & 0.0 & 0.0 & 0.0 & $\mathbf{9 1 . 6}$ & 2.8 & 5.6 \\
Clap & 1.4 & 0.0 & 0.0 & 6.3 & $\mathbf{9 2 . 4}$ & 0.0 \\
Wave & 0.0 & 0.0 & 0.0 & 7.6 & 6.9 & $\mathbf{8 5 . 4}$ \\
\hline
\end{tabular}

\subsection{Performance on combined features}

From the previous subsection, we found that different features had different powers in distinguishing classes of action. In order to overcome their own limitations, we combine the MGD (derived from the MHH) and the histogram of the MHI, by concatenation. Table 7 shows the confusion matrix obtained from our system when these combined features were used. From this table, we can see that the overall performance has a significant improvement over Ke's method, which is based on volumetric features. Note that a good performance, averaging over $80 \%$ in correct classifications, is achieved in distinguishing the six actions in this challenging dataset.

We compared our results with other methods on this challenging dataset and we summarize the correctly classified rates in Table 8 . From this table, we can see that MHH has made a significant improvement in comparison with MHI. Furthermore, the MGD feature gives a better performance than the MHH itself. The best performance, which gives significantly better classification results, came from the combined feature, which is based on the histogram of the MHI and the MGD. 
Table 7

MGD \& Hist. of MHI's confusion matrix, trace $=481.9$, mean performance $=80.3 \%$

\begin{tabular}{ccccccc} 
& Walk & Jog & Run & Box & Clap & Wave \\
\hline Walk & $\mathbf{6 6 . 0}$ & 31.3 & 0.0 & 0.0 & 2.1 & 0.7 \\
Jog & 13.9 & $\mathbf{6 2 . 5}$ & 21.5 & 1.4 & 0.0 & 0.7 \\
Run & 2.1 & 16.7 & $\mathbf{7 9 . 9}$ & 0.0 & 0.0 & 1.4 \\
Box & 0.0 & 0.0 & 0.0 & $\mathbf{8 8 . 8}$ & 2.8 & 8.4 \\
Clap & 0.0 & 0.0 & 0.0 & 3.5 & $\mathbf{9 3 . 1}$ & 3.5 \\
Wave & 0.0 & 0.0 & 0.0 & 1.4 & 6.9 & $\mathbf{9 1 . 7}$ \\
\hline
\end{tabular}

Table 8

Overall correctly classified rate (\%) for all the methods on this open, challenging dataset.

\begin{tabular}{lc}
\hline Method & Rate(\%) \\
\hline SVM on local features (Schuldt et al. (2004))* & 71.7 \\
Cascade of filters on volumetric features (Ke et al. (2005)) & 63 \\
SVM on MHI (Meng et al. (2006b)) & 63.5 \\
SVM_2K on MHI \& MMHI (Meng et al. (2006a)) & 65.3 \\
SVM on MHH_S & 69.6 \\
SVM on MGD & 72.1 \\
SVM on HWT of MHI \& Hist. of MHI (Meng et al. (2007b)) & 70.9 \\
SVM on MGD \& Hist. of MHI & $\mathbf{8 0 . 3}$ \\
\hline
\end{tabular}

It should be mentioned here that some performance results presented in the literature (Dollár et al. (2005); Niebles et al. (2006); Yeo et al. (2006)) are quoted as higher than ours, when performing classification experiments on (parts of) this public dataset. However, we have not included these in Table 8 , because they are not directly comparable to ours, either because they have omitted to use all of the data, or because they have performed an easier classification task, or both. For example, Dollár et al. (2005) achieved a correct classification rate of $81.2 \%$, but the evaluation omitted scenarios s2 and s4. Our earlier results, shown in Fig. 8, clearly indicate that scenario s2 is the most difficult subset. This is also witnessed in the results of our best system (MGD and histogram of $\mathrm{MHI}$ ), which give a mean performance of $80.4 \%, 63.5 \%$, $82.4 \%$ and $87 \%$ for the four scenarios s1, s2, s3 and s4 respectively. When we combine data from the s1 and s3 subsets, as used by Dollár et al. (2005), and re-train our SVM classifiers on this combined data set, then we obtain a mean performance of $82.8 \%$, which is slightly better than the result of Dollár et al. (2005) (81.2\%). Niebles et al. (2006) obtained similar results with $81.5 \%$ and 
Yeo et al. (2006) obtained 86.0\%, but they did an easier task of classifying each complete sequence (containing 4 repetitions of same action) into one of six classes, while our method was trained the same way as other papers(Ke et al. (2005); Meng et al. (2006b,a, 2007b)), that is to detect a single instance of each action, within arbitrary sequences in the dataset. Furthermore, Yeo et al. (2006) did not use the difficult subset 2 of the dataset, as was the case in Dollár et al. (2005).

\section{Conclusion and discussions}

In this paper, we have proposed new, descriptive representations for human action recognition systems, which may easily be implemented in an embedded computer vision context. The proposed method does not rely on accurate tracking as many other works do, since most tracking algorithms incur an extra computational cost for the system. Our system is based on simple 'temporal template' features in order to achieve high-speed recognition in real-time embedded applications.

In order to improve the state of the art performance in temporal template based systems, we have proposed a new representation for motion information in video and this is called the Motion History Histogram (MHH). The representation extends previous work on temporal template (MHI related) representations by additionally storing frequency information as the number of times motion is detected at every pixel, further categorized into the length of each motion. In essence, maintaining the number of contiguous motion frames removes a significant limitation of MHI, which only encodes the time from the last observed motion at every pixel. It can be used either independently, or combined with information derived from the MHI, to give human action recognition systems with improved performance over existing comparable temporal template based systems.

In terms of our work on combining features from MHI and MHH, we acknowledge that we have not generated a provably optimal feature combination. Rather, we have selected two of the most promising feature types that we have tested, that make explicit different aspects of the motion sequence, and we have shown that the combined performance is better than either feature performance alone. This suggests that further fruitful research would be to search for optimal combinations of temporal-template type features, for example using a genetic algorithm, which is well suited to such a task.

In comparison with local SVM methods of Schuldt et al. (2004) and a cascade of filters on volumetric features of Ke et al. (2005), our feature vectors are computationally inexpensive. Even though we don't use a validation dataset 
for parameter tuning in SVM training, we have demonstrated a significant improvement in recognition performance over other methods tested in a comparable rigorous way on the large, public human action database (Schuldt et al. (2004)).

\section{References}

Aggarwal, J. K., Cai, Q., 1999. Human motion analysis: a review. Comput. Vis. Image Underst. 73 (3), 428-440.

Blank, M., Gorelick, L., Shechtman, E., Irani, M., Basri, R., 2005. Actions as spacetime shapes. In: ICCV. pp. 1395-1402.

Bobick, A. F., Davis, J. W., 2001. The recognition of human movement using temporal templates. IEEE Trans. Pattern Anal. Mach. Intell. 23 (3), 257-267.

Bradski, G. R., Davis, J. W., 2002. Motion segmentation and pose recognition with motion history gradients. Mach. Vis. Appl. 13 (3), 174-184.

Campbell, L. W., Bobick, A. F., 1995. Recognition of human body motion using phase space constraints. In: ICCV. pp. 624-630.

Cristianini, N., Shawe-Taylor, J., 2000. An Introduction to Support Vector Machines (and other kernel-based learning methods). Cambridge University Press, Cambridge, UK.

Dalal, N., Triggs, B., Schmid, C., 2006. Human detection using oriented histograms of flow and appearance. In: ECCV (2). pp. 428-441.

Davis, J. W., 2001. Hierarchical motion history images for recognizing human motion. In: IEEE Workshop on Detection and Recognition of Events in Video. pp. $39-46$.

Davis, J. W., Tyagi, A., 2006. Minimal-latency human action recognition using reliable-inference. Image Vision Comput. 24 (5), 455-472.

Dollár, P., Rabaud, V., Cottrell, G., Belongie, S., October 2005. Behavior recognition via sparse spatio-temporal features. In: VS-PETS.

Efros, A. A., Berg, A. C., Mori, G., Malik, J., 2003. Recognizing action at a distance. In: ICCV. pp. 726-733.

Farmer, J., Casdagli, M., Eubank, S., Gibson, J., 1991. State-space reconstruction in the presence of noise. Physics D 51, 52-98.

Gao, J., Hauptmann, A. G., Bharucha, A., Wactlar, H. D., 2004. Dining activity analysis using a hidden markov model. In: ICPR (2). pp. 915-918.

Green, R. D., Guan, L., 2004. Quantifying and recognizing human movement patterns from monocular video images-part ii: applications to biometrics. IEEE Trans. Circuits Syst. Video Techn. 14 (2), 191-198.

Hastie, T., Rosset, S., Tibshirani, R., Zhu, J., 2004. The entire regularization path for the support vector machine.

URL citeseer.ist.psu.edu/hastie04entire.html

Joachims, T., 1999. Making large-scale SVM learning practical. In: Advances in Kernel Methods - Support Vector Learning. MIT-Press, USA, oikonomopoulos, Antonios and Patras, Ioannis and Pantic, Maja eds.

URL http://svmlight.joachims.org/ 
Ke, Y., Sukthankar, R., Hebert., M., 2005. Efficient visual event detection using volumetric features. In: ICCV. pp. 166-173, beijing, China, Oct. 15-21, 2005.

Meng, H., Freeman, M., Pears, N., Bailey, C., 2008. Real-time human action recognition on an embedded, reconfigurable video processing architecture. Journal of Real-Time Image Processing.

Meng, H., Pears, N., Bailey, C., 2006a. Human action classification using SVM_2K classifier on motion features. In: LNCS. Vol. 4105. Istanbul, Turkey, pp. 458-465.

Meng, H., Pears, N., Bailey, C., 2006b. Recognizing human actions based on motion information and SVM. In: 2nd IET International Conference on Intelligent Environments. IET, Athens, Greece, pp. 239-245.

Meng, H., Pears, N., Bailey, C., 2007a. A human action recognition system for embedded computer vision application. In: CVPR workshop on Embeded Computer Vision.

Meng, H., Pears, N., Bailey, C., March 2007b. Motion information combination for fast human action recognition. In: 2nd International Conference on Computer Vision Theory and Applications (VISAPP07). Barcelona, Spain.

Moeslund, T., Hilton, A., Kruger, V., November 2006. A survey of advances in vision-based human motion capture and analysis. Comput. Vis. Image Underst. 103 (2-3), 90-126.

Nascimento, J. C., Figueiredo, M. A. T., Marques, J. S., 2005. Recognition of human activities using space dependent switched dynamical systems. In: IEEE Int. Conf. on Image Processing, ICIP.

Niebles, J., Wang, H., Fei-Fei, L., 2006. Unsupervised learning of human action categories using spatial-temporal words. In: BMVC06. p. III:1249.

Ogata, T., Tan, J. K., Ishikawa, S., 2006. High-speed human motion recognition based on a motion history image and an eigenspace. IEICE Transactions on Information and Systems E89 (1), 281-289.

Oikonomopoulos, A., Patras, I., Pantic, M., June 2006. Kernel-based recognition of human actions using spatiotemporal salient points. In: Proceedings of CVPR workshop 06. Vol. 3. pp. 151-156.

URL http://pubs.doc.ic.ac.uk/Pantic-CVPR06-1/

Schuldt, C., Laptev, I., Caputo, B., 2004. Recognizing human actions: a local SVM approach. In: ICPR. Cambridge, U.K.

Stauffer, C., Grimson, W. E. L., 2000. Learning patterns of activity using real-time tracking. IEEE Transactions on Pattern Analysis and Machine Intelligence 22 (8), $747-757$.

URL citeseer.ist.psu.edu/stauffer00learning.html

Weinland, D., Ronfard, R., Boyer, E., 2005. Motion history volumes for free viewpoint action recognition. In: IEEE International Workshop on modeling People and Human Interaction (PHI'05).

URL http://perception.inrialpes.fr/Publications/2005/WRB05

Wong, S.-F., Cipolla, R., 2005. Real-time adaptive hand motion recognition using a sparse bayesian classifier. In: ICCV-HCI. pp. 170-179.

Wong, S.-F., Cipolla, R., 2006. Continuous gesture recognition using a sparse bayesian classifier. In: ICPR (1). pp. 1084-1087.

Yeo, C., Ahammad, P., Ramchandran, K., Sastry, S., 2006. Compressed domain real-time action recognition. In: IEEE International Workshop on Multimedia 
Signal Processing (MMSP) - 2006. IEEE, Washington, DC, USA. 